Pacific Journal of Mathematics

EQUATIONAL CLASSES OF MODULAR LATTICES 


\title{
EQUATIONAL CLASSES OF MODULAR LATTICES
}

\author{
KIRBY A. BAKER
}

\begin{abstract}
One natural question of lattice theory has been (i) whether there exists an equational class of lattices which cannot be characterized by any finite list of lattice identities. Another question, due to $B$. Jónsson, is (ii) whether there exists an equational class of lattices which is not determined by its finite members. We shall show that the answers to both questions are affirmative, even with the additional requirement of modularity. The examples are constructed from lattices corresponding to projective planes.
\end{abstract}

Using different methods, R. McKenzie [9] has independently answered the first question (without modularity).

1. Stable classes of lattices. By an equational class of lattices is meant the class of all lattices satisfying some fixed finite or infinite set of lattice identities. Birkhoff [2] has shown for abstract algebras in general that a class of algebras with the "same" operations is an equational class if and only if it is closed under the formation of direct products, subalgebras, and homomorphic images. Jónsson [7] has sharpened this result in the case of algebras whose lattices of congruence relations are distributive; we shall merely state his key lemma, for the case of lattices. If $\mathscr{K}$ is any class of lattices, let $\mathscr{K}^{e}$ be the equational class of lattices generated by $\mathscr{K}$, i.e., the class of all lattices satisfying all lattice identities true in all lattices of $\mathscr{K}$. For further terminology, see Birkhoff [3].

Lemma 1.1. (Jónsson [7], Corollary 3.2). If $\mathscr{K}^{-}$is a class of lattices and if a subdirectly irreducible lattice $L$ is in $\mathscr{K}^{e}$, then $L$ is a homomorphic image of a sublattice of an ultraproduct of lattices from $\mathscr{K}$.

The following concept will be useful.

Definition 1.2. A class $\mathscr{K}$ of lattices is stable if $\mathscr{K}$ is closed under the formation of sublattices, homomorphic images, and ultraproducts.

Clearly, any equational class of lattices is stable. Less trivial examples are provided by the following fact.

LEMmA 1.3. Let $P$ be a finite partially ordered set, and let 
$\mathscr{N}(P)$ be the class of lattices which do not have any subset isomorphic to $P$. Then $\mathcal{N}(P)$ is a stable class of lattices.

Proof. $\mathscr{N}(P)$ is clearly closed under the formation of sublattices. Since the property of not containing a copy of $P$ can be expressed by a finite sentence, $\mathscr{N}(P)$ is closed under the formation of ultraproducts. Finally, it is easy to show that if a homomorphic image of a lattice $L$ contains a copy of $P$, then $L$ itself contains a copy of $P$.

We list and then prove several properties of stable classes.

Property 1.4. If $\mathscr{K}$ is a stable class of lattices and $L \in \mathscr{K}^{e}$ is subdirectly irreducible, then $L \in \mathscr{K}$.

Property 1.5. If $\mathscr{K}$ is a stable class of lattices, then $\mathscr{K}^{e}$ consists of subdirect products of lattices from $\mathscr{K}$.

Property 1.6. If $\mathscr{K}_{1}$ and $\mathscr{K}_{2}$ are stable classes of lattices, then $\mathscr{K}_{1}^{e}=\mathscr{K}_{2}^{e}$ if and only if $\mathscr{K}_{1}$ and $\mathscr{K}_{2}$ have the same subdirectly irreducible members.

Property 1.7. Arbitrary intersections and finite unions of stable classes are stable.

Proofs. Property 1.4 is a direct consequence of Lemma 1.1. For Property 1.5, first note that any subdirect product of lattices from $\mathscr{K}^{r}$ is in $\mathscr{K}^{e}$. Conversely, if $L$ is in $\mathscr{K}^{e}$, a theorem of Birkhoff [3, Th. 15, p. 193] states that $L$ can be represented as a subdirect product of subdirectly irreducible lattices $L_{\alpha}$. Since the $L_{\alpha}$ are homomorphic images of $L$, we have $L_{\alpha} \in \mathscr{K}^{e}$, and hence $L_{\alpha} \in \mathscr{K}$ by Property 1.4. Property 1.6 follows from Property 1.4 and the fact that an equational class is uniquely determined by its subdirectly irreducible members. Property 1.7 is readily verified.

We shall frequently use the following fact.

Lemma 1.8. Let $\mathscr{K}$ be a stable class of lattices, let $\left\{P_{\alpha}\right\}$ be any collection of finite partially ordered sets, and let $\mathscr{K}^{\prime \prime}$ be the class of lattices in $\mathscr{K}$ which contain no $P_{\alpha}$ as a partially ordered subset. Then $\mathscr{K}^{\prime}$ is stable.

Proof. Apply Lemma 1.3 and Property 1.7 to $\mathscr{K} \cap\left(\bigcap_{\alpha} \mathscr{N}^{-}\left(P_{\alpha}\right)\right)$. It should be remarked that stable classes are type classes [4, p. 214], and thus the stable classes can be regarded as forming a set.

2. Weak projective planes. By a "projective plane," or, for 
emphasis, a nondegenerate projective plane, we shall mean the lattice of subspaces of a projective plane in the ordinary sense [6, p. 7]. More generally, by a weak projective plane we shall mean any sublattice of a nondegenerate projective plane. A weak projective plane which is not nondegenerate will of course be called degenerate. Such a lattice can still be regarded as a partial plane [cf. 6, p. 9].

It should be noted that any nondegenerate projective plane is subdirectly irreducible (in fact, simple) and that any sublattice of a degenerate weak projective plane is again degenerate.

Lemma 2.1. Weak projective planes constitute a stable class of lattices.

Proof. Weak projective planes can be shown to be just the modular lattices containing no five-element chain [cf. 6, p. 9]. Since modular lattices constitute an equational class, the result follows from Lemma 1.8 (with a single $P_{\alpha}$, consisting of a five-element chain).

We now define Desarguesian weak projective planes and show that they form a stable class; Pappian weak projective planes are similar.

Definition 2.2. An anti-Desarguesian partially ordered set $D$ is a partially ordered set corresponding to a configuration which represents a failure of Desargues' theorem in a projective plane. Specifically, $D$ represents two triangles perspective from a point but not from a line and includes the lines of the triangle formed by the three points of the configuration which fail to be collinear. A weak projective plane is Desarguesian if it has no subset isomorphic to an antiDesarguesian partially ordered set.

Because some points of an anti-Desarguesian configuration may or may not lie on various lines of the configuration, there is more than one anti-Desarguesian partially ordered set. The above definition of a Desarguesian weak projective plane of course reduces to the usual one in the case of nondegenerate projective planes. On the other hand, any degenerate weak projective plane is Desarguesian.

Lemma 2.3. The Desarguesian weak projective planes form a stable class of lattices.

Proof. Lemma 1.8.

Similarly, for Pappus' theorem in place of Desargues', we can define anti-Pappian partially ordered sets and Pappian weak projective planes and obtain 
class of lattices.

REMARK. Schützenberger [10] and Jónsson [8] have actually given single lattice identities which can be used to characterize Desarguesian projective planes among all projective planes.

Lemma 2.5. Any Pappian weak projective plane is Desarguesian, and any finite Desarguesian weak projective plane is Pappian.

Proof. This is known for nondegenerate projective planes: The Desarguesian projective planes are those which can be coordinatized by a skew field, and the Pappian projective planes are those which can be coordinatized by a field [1, pp. 73-75]. As remarked above, the degenerate weak projective planes are always Desarguesian, and similarly, always Pappian.

LEMma 2.6. Let $\mathscr{D}$ be the equational class generated by the Desarguesian weak projective planes, and let $\mathscr{P}$ be the equational class generated by the Pappian weak projective planes. Then $\mathscr{D}$ and $\mathscr{P}$ have the same finite members but are not identical.

Proof. By Property 1.5 combined with Lemmas 2.3 and 2.4, the finite lattices in $\mathscr{D}$ and $\mathscr{P}$ are subdirect products of finite Desarguesian and Pappian weak projective planes, respectively. But by Lemma 2.5, finite Desarguesian and Pappian weak projective planes are the same. To show that $\mathscr{D}$ and $\mathscr{P}$ are not identical, it suffices by Property 1.6 to exhibit a subdirectly irreducible Desarguesian weak projective plane which is not Pappian. Any projective plane coordinatized by a noncommutative skew field provides such an example.

THEOREM 2.7. There exists an equational class of modular lattices which is not determined by its finite members.

Proof. $\mathscr{D}$ of Lemma 2.6 is such an equational class.

REMARK. If $\mathscr{R}$ is the equational class generated by the rational projective plane, i.e., the lattice of subspaces of a three-dimensional vector space over the rational numbers, then it can be shown that $\mathscr{R}$ also is not determined by its finite members. In fact, all finite members of $\mathscr{R}$ are degenerate, and the class of degenerate planes is stable.

3. Sublattices of the lattice of equational classes. Let $\Lambda$ (respectively, $M$ ) be the lattice of equational classes of lattices (respec- 
tively, of modular lattices). Jónsson [7, Corollary 4.1] has shown that $A$ is distributive; hence $M$ is also. Not much is known about the nature of the sublattices of $\Lambda$ and $M$. The following fact is therefore of interest.

THEOREM 3.1. $M$ has a complete sublattice isomorphic to the Boolean lattice of all subsets of a countable set.

The proof of Theorem 3.1, given below, will depend on the following definitions and lemma.

For each integer prime $q$, let $P_{q}$ be the Desarguesian projective plane of order $q$, i.e., the lattice of subspaces of a three-dimensional vector space over the Galois field $G F(q)$ with $q$ elements. Since a nondegenerate Desarguesian projective plane can be coordinatized by a skew field, every such plane has an associated characteristic which is either 0 or a finite prime. Thus the characteristic of $P_{q}$ is $q$. Let $\mathscr{N}\left(P_{q}\right)$ be the stable class of Lemma 1.3, with $\mathscr{N}\left(P_{q}\right)^{e}$ the equational class it generates.

LEMma 3.2. If $Q$ is any Desarguesian weak projective plane, then $Q \in \mathscr{N}\left(P_{q}\right)$ if and only if $Q$ is either degenerate or of a characteristic different from $q$.

Proof. If $Q \notin \mathscr{N}\left(P_{q}\right)$, i.e., if $Q$ does contain a copy of $P_{q}$ as a subset, then $Q$ must (for lack of room) contain $P_{q}$ as a sublattice. Hence $Q$ is not degenerate, and the characteristic of $Q$ must be $q$. On the other hand, if $Q$ is a nondegenerate projective plane of characteristic $q$, then $Q$ does have a subplane, hence a subset, isomorphic to $P_{q}$. Thus $Q \notin \mathscr{N}\left(P_{q}\right)$.

Proof of Theorem 3.1. We take the countable set to be the set $I$ of finite integer primes. We define for each subset $S$ of $\Pi$ an equational class $\mu(S) \in M$, as follows. Let $\mu(S)=\mathscr{D} \cap\left(\bigcap_{q \notin S} \mathscr{N}\left(P_{q}\right)^{e}\right)$, where (as before) $\mathscr{D}$ is the equational class generated by the Desarguesian weak projective planes. By Lemma 3.2 and Property 1.4, the subdirectly irreducible lattices in $\mu(S)$ are precisely those which are Desarguesian weak projective planes and are either degenerate, of characteristic 0 , or of a characteristic in $S$. Thus the equational classes $\mu(S)$ are different for different sets $S$, i.e., $\mu$ is one-to-one.

To complete the proof, we must show that $\mu$ is a complete lattice homomorphism. Accordingly, let $\left\{S_{\alpha}: \alpha \in A\right\}$ be a collection of subsets of $\Pi$. We must verify that (i) $\mu\left(\bigcap_{\alpha} S_{\alpha}\right)=\bigcap_{\alpha} \mu\left(S_{\alpha}\right)$ and that (ii) $\mu\left(\mathrm{U}_{\alpha} S_{\alpha}\right)=\mathrm{V}_{\alpha} \mu\left(S_{\alpha}\right)$. The property (i) is immediate from the definition of $\mu$. For (ii), the inclusion $\supseteq$ follows from the isotonicity of $\mu$, and the inclusion $\subseteq$ can be checked by examining characteristics of the 
subdirectly irreducible elements of $\mu\left(\bigcup_{\alpha} S_{\alpha}\right)$. (If the index set $A$ is empty, $\bigcap_{\alpha} \mu\left(S_{\alpha}\right)$ must be interpreted to be $\mathscr{D}$, and $\bigcup_{\alpha} \mu\left(S_{\alpha}\right)$ must be $\mu(\varnothing$.$) The proof of Theorem 3.1$ is thus complete.

CoRollary 3.3. The lattice $M$ of equational classes of modular lattices and the lattice $\Lambda$ of all equational classes of lattices both have the cardinality of the continuum.

The question of determining the cardinality of $\Lambda$ was suggested to the author by G. Grätzer. The cardinality of $\Lambda$ has also been determined independently by R. McKenzie [9].

4. Axioms for equational classes. A set of lattice identities used to define an equational class is called a set of axioms for that class. Some equational classes of lattices, for example the class of modular lattices, can be defined by only a single axiom, in addition to the identities which define the concept of a lattice. Moreover, it is wellknown that any finite set of axioms for an equational class of lattices can be replaced by a single axiom. A natural question then arises: Can every equational class of lattices be defined by a single axiom?

By Corollary 3.3, the answer to this question is evidently negative, since there are only countably many lattice identities. Theorem 4.2 below will give an explicit example of an equational class of lattices which can be defined only by infinitely many axioms. Although the derivation could be given in terms of the lattice of all theories of lattices, we shall work within the lattice of equational classes.

Recall that an element $x$ of a complete lattice $L$ is compact [3, p. 186] if whenever $x \leqq \mathrm{~V}_{\alpha} y_{\alpha}$ for some family of $y_{\alpha} \in L$, then $x \leqq y_{\alpha(1)} \vee \cdots \vee y_{\alpha(n)}$ for some finite set of indices $\alpha(1), \cdots, \alpha(n)$. If $x$ satisfies the dual condition instead, we shall say that $x$ is cocompact. A complete $L$ is algebraic [3, p. 187] if every element of $L$ is a join of compact elements; $L$ will be termed coalgebraic if its dual is algebraic.

LEMmA 4.1. Let $\Lambda$ be the lattice of equational classes of lattices. Then 1 is a coalgebraic distributive lattice in which the cocompact elements are those equational classes which have a finite set of axioms.

Proof. Jónsson has shown that $\Lambda$ is distributive [7, Corollary 4.1]. Furthermore, Jónsson and Engeler [7, §4] have made the following observation: If $F$ is a free lattice on infinitely many generators, if $F^{\prime}$ is $F$ with the endomorphisms of $F$ included as unary operations, and if $\Theta\left(F^{\prime}\right)$ is the lattice of congruence relations on $F^{\prime}$, then $\Lambda$ is 
dually isomorphic to $\Theta\left(F^{\prime}\right)$. Under this dual isomorphism, the equational class generated by a single lattice identity $f\left(x_{1}, \cdots, x_{n}\right)=g\left(x_{1}, \cdots, x_{n}\right)$ corresponds to the smallest congruence relation $\theta(f, g)$ on $F^{\prime}$ identifying $f$ and $g$, regarded now as elements of $F^{\prime}$. But $\Theta\left(F^{\prime}\right)$ is algebraic, with compact elements being precisely the finite joins of elements of the form $\theta(f, g)[5, \mathrm{p} .11]$. Via the dual isomorphism, the assertion of the lemma follows.

THEOREM 4.2. Let $\mathscr{E}$ be the equational class of modular lattices generated by the Desarguesian weak projective planes which are either degenerate or of characteristic 0 . Then $\mathscr{E}$ has no finite set of axioms.

Proof. In the notation of the proof of Theorem 3.1, $\mathscr{E}=u(\varnothing)$. Since $\varnothing$ is not cocompact in the lattice of subsets of the infinite set $\Pi, \mathscr{E}$ is not cocompact in $A$.

Added in proof. Still another equational class not generated by its finite members has been announced by $\mathrm{R}$. Wille [Notices of the Amer. Math. Soc. 15 (August, 1968), p. 781].

\section{REFERENCES}

1. E. Artin, Geometric algebra, Interscience, New York, 1957.

2. G. Birkhoff, On the structure of abstract algebras, Proc. Cambridge Phil. Soc. 31 (1935), 433-454.

3. - Lattice theory, 3rd. ed., Amer. Math. Soc. Colloq. Publ. 25, Providence, 1967.

4. P. M. Cohn, Universal algebra, Harper and Row, New York, 1965.

5. R. P. Dilworth, Structure and decomposition theory of lattices, Proc. Symp. in Pure Math. II, Amer. Math. Soc., Providence, 1961.

6. M. Hall, Jr., Projective planes and related topics, Calif. Inst. of Technology, 1954.

7. B. Jónsson, Algebras whose congruence lattices are distributive, Math. Scand. (to appear)

8. Modular lattices and Desargues' theorem, Math. Scand. 2 (1954), 295-314.

9. R. McKenzie, On equational theories of lattices (to appear).

10. M. Schützenberger, Sur certains axioms de la théorie des structures, C. R. Acad. Sci. Paris 221 (1945), 218-220.

Received May 16, 1968.

California Institute of Technology

UNIVERSITY OF CALIFORNIA AT LOS ANGELES 



\section{PACIFIC JOURNAL OF MATHEMATICS}

\section{EDITORS}

H. ROYDEN

Stanford University

Stanford, California

R. R Phelps

University of Washington

Seattle, Washington 98105
J. DUGUNDJI

Department of Mathematics

University of Southern California

Los Angeles, California 90007

RICHARD ARENS

University of California

Los Angeles, California 90024

\section{ASSOCIATE EDITORS}
E. F. BECKENBACH
B. H. NeumanN
F. WOLF
K. YosidA

\section{SUPPORTING INSTITUTIONS}

UNIVERSITY OF BRITISH COLUMBIA

CALIFORNIA INSTITUTE OF TECHNOLOGY

UNIVERSITY OF CALIFORNIA

MONTANA STATE UNIVERSITY

UNIVERSITY OF NEVADA

NEW MEXICO STATE UNIVERSITY

OREGON STATE UNIVERSITY

UNIVERSITY OF OREGON

OSAKA UNIVERSITY

UNIVERSITY OF SOUTHERN CALIFORNIA
STANFORD UNIVERSITY

UNIVERSITY OF TOKYO

UNIVERSITY OF UTAH

WASHINGTON STATE UNIVERSITY

UNIVERSITY OF WASHINGTON

AMERICAN MATHEMATICAL SOCIETY CHEVRON RESEARCH CORPORATION TRW SYSTEMS

NAVAL WEAPONS CENTER 


\section{Pacific Journal of Mathematics

Vol. 28, No. $1 \quad$ March, 1969

Patrick Robert Ahern, On the geometry of the unit ball in the space of real annihilating measures .............................. 1

Kirby Alan Baker, Equational classes of modular lattices ............. 9

E. F. Beckenbach and Gerald Andrew Hutchison, Meromorphic minimal surfaces ......................................... 17

Tae Ho Choe, Intrinsic topologies in a topological lattice ..............

John Bligh Conway, A theorem on sequential convergence of measures and

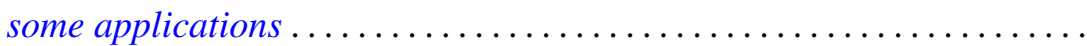

Roger Cuppens, On the decomposition of infinitely divisible probability laws without normal factor.

Lynn Harry Erbe, Nonoscillatory solutions of second order nonlinear

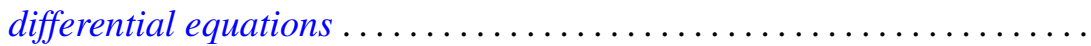

Burton I. Fein, The Schur index for projective representations of finite

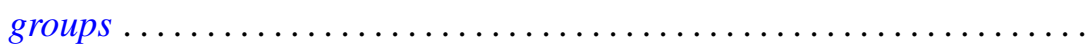

Stanley P. Gudder, A note on proposition observables............... 101

Kenneth Kapp, On Croisot's theory of decompositions ............... 105

Robert P. Kaufman, Gap series and an example to Malliavin's theorem . . . 117

E. J. McShane, Robert Breckenridge Warfield, Jr. and V. M. Warfield,

Invariant extensions of linear functionals, with applications to measures and stochastic processes ................

Marvin Victor Mielke, Rearrangement of spherical modifications ...

Akio Osada, On unicity of capacity functions ..............

Donald Steven Passman, Some 5/2 transitive permutation groups ...

Harold L. Peterson, Jr., Regular and irregular measures on groups and dyadic spaces...

Habib Salehi, On interpolation of $q$-variate stationary stochastic processes...

Michael Samuel Skaff, Vector valued Orlicz spaces generalized

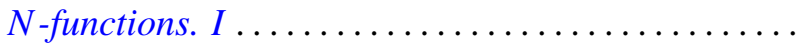

A. J. Ward, On $H$-equivalence of uniformities. II...........

Thomas Paul Whaley, Algebras satisfying the descending chain condition for subalgebras...

G. K. White, On subgroups of fixed index

Martin Michael Zuckerman, A unifying condition for implications among the axioms of choice for finite sets ................. 